\title{
colonnes ballastées \\ essais de chargement \\ et calculs par la méthode des éléments finis
}

\author{
par \\ Michel Morgenthaler \\ Ingénieur à SIF-Entreprise Bachy \\ Bernard Cambou \\ Maître Assistant à l'École Centrale Lyonnaise \\ et \\ Guy Sanglerat \\ Directeur du Service Interrégional \\ Sol et Fondation à Socotec
}

\section{Circonstances de l'essai}

\subsection{Lieu, date et intervenants}

L'hypermarché Chamnord, près de Chambéry, a été fondé sur un terrain préalablement traité par Vibroflottation et Colonnes Ballastées. Les travaux ont débuté en 1976, avec les sociétés Betrec pour l'ingéniérie, Geoprojets pour I'étude géotechnique, SIF-Bachy pour le traitement du sol et Fougerolle pour le gros-œuvre. L'opération a été effectuée sous le contrôle de Socotec.

\section{2 Éléments descriptifs du projet}

Il s'agit d'un bâtiment à ossature en béton armé avec poutres précontraintes de $12 \mathrm{~m}$ de portée, couvrant $22000 \mathrm{~m}^{2}$. Les poteaux descendent des charges de $160 \mathrm{kN}$ à $3870 \mathrm{kN}$. La contrainte sous les dallages est de $10 \mathrm{kPa}$.

Les reconnaissances préliminaires ont permis de distinguer deux zones (fig. 1).

Zone I: $25 \%$ de la surface construite, présentant des graves argileuses affleurantes, ne nécessitant qu'une homogénéisation et quelques colonnes ballastées standard dans les zones faibles décelées par un contrôle, appui par appui, au pénétromètre Andina.

Zone II : $75 \%$ de la surface construite, présentant $4 \mathrm{~m}$ de limons mous au-dessus des graves argileuses, nécessitant:

- sous les appuis : des colonnes ballastées cimentées en tête sur la hauteur des limons, ancrées dans les graves argileuses compactées par vibration.

- sous les dallages : des colonnes ballastées standard de 4 à $6 \mathrm{~m}$ de profondeur, réparties selon une maille de $3 \times 3 \mathrm{~m}$

SIF-Bachy a réalisé $12900 \mathrm{~m}$ de colonnes ballastées, dont $3100 \mathrm{~m}$ avec cimentation, en appliquant le procédé Keller. Le traitement de sol effectué illustre bien les possibilités offertes par les colonnes ballastées. Dans le cas présent, la solution a été retenue parce que, tout en présentant une sécurité suffisante, elle était plus économique que les solutions concurrentes: pieux profonds, préchargement, radier général.

\subsection{Pourquoi des essais et des recherches}

L'objet principal de cet article est de présenter les essais de chargement in situ qui ont été réalisés à l'occasion du chantier et les calculs par la méthode des éléments finis qui ont été effectués en parallèle.

Cette recherche a été conduite conjointement par la société SIF-Bachy, I'École Centrale Lyonnaise et Socotec.

On notera le caractère de rareté des essais de chargement grandeur nature, surtout appliqués à des colonnes ballastées, et l'originalité de la simulation théorique menée parallèlement.

\section{Rappels sur la vibroflottation et les colonnes ballastées}

\subsection{La vibroflottation, son principe}

"Vibroflottation » désigne, par un terme anglais accepté dans le vocabulaire technologique français, la technique qui consiste à traiter les sols par vibration dans la masse au moyen d'un vibreur introduit dans le terrain. A une homothétie près, le procédé est relativement analogue à la vibration du béton au moyen d'une aiguille vibrante.

II est essentiel de souligner que le traitement vibratoire par " vibroflottation » s'effectue par définition dans la masse de la zone à traiter, puisque ce caractère le démarque fondamentalement d'autres procédés de traitement par des vibrations ou des chocs engendrés depuis la surface du terrain, tels que le compactage par rouleau vibrant (vibration entretenue) ou le compactage dynamique (chocs répétés d'une masse tombant en chute libre).

La vibroflottation permet donc une action contrôlée et homogène en profondeur, quelle que soit la profondeur (aux limites du matériel près).

La vibroflottation se suffit à elle-même dans les sols purement pulvérulents : sables, graviers, galets. Dans de tels sols, dont les particules sont indépendantes et soumises aux seules forces de gravité, de contact intergranulaire et à la pression interstitielle, la vibration modifie temporairement l'équilibre de ces forces et provoque un nouvel arrangement du squelette, qui devient 
ZONE 2

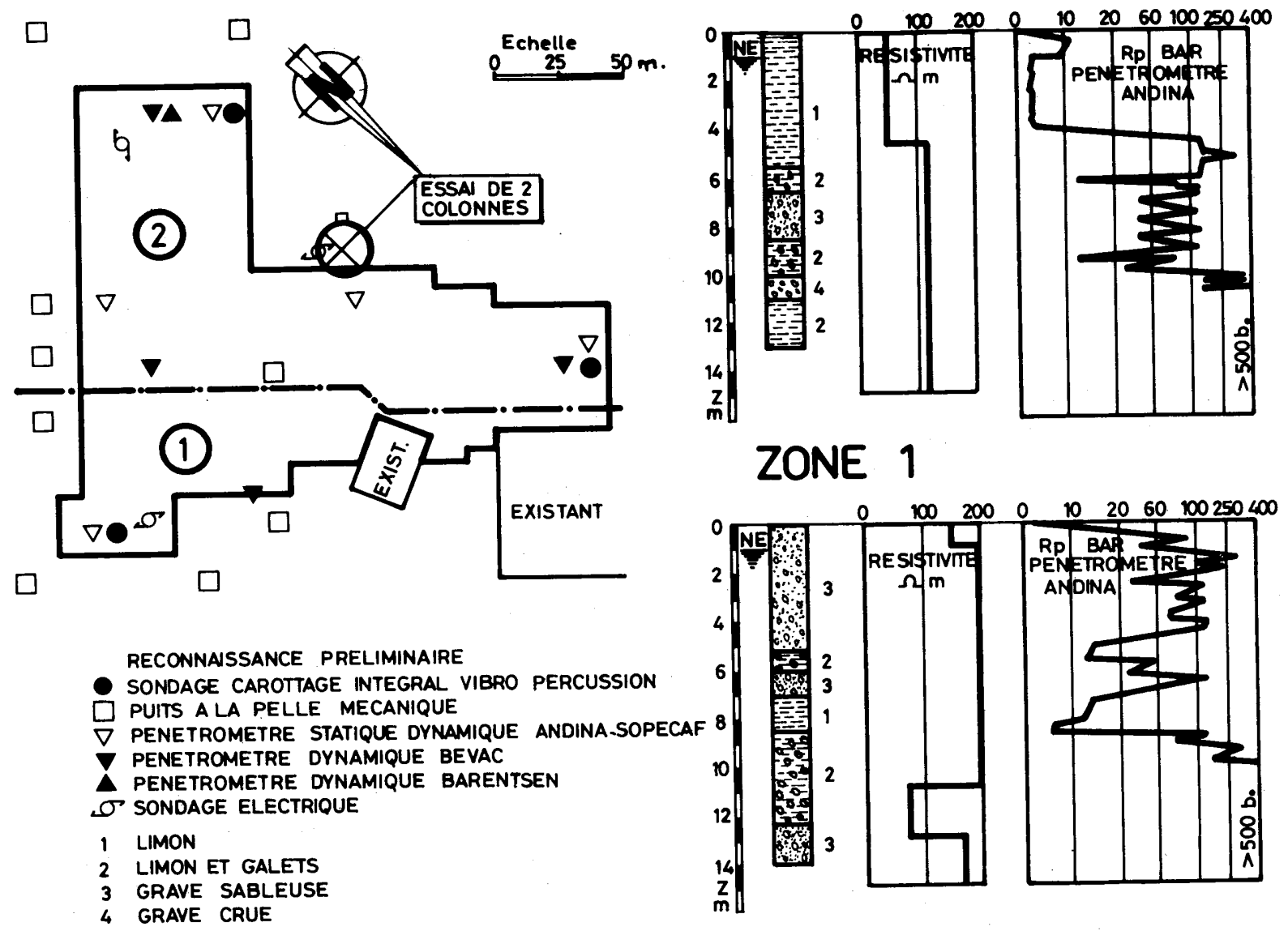

Fig. 1

Fig. 2

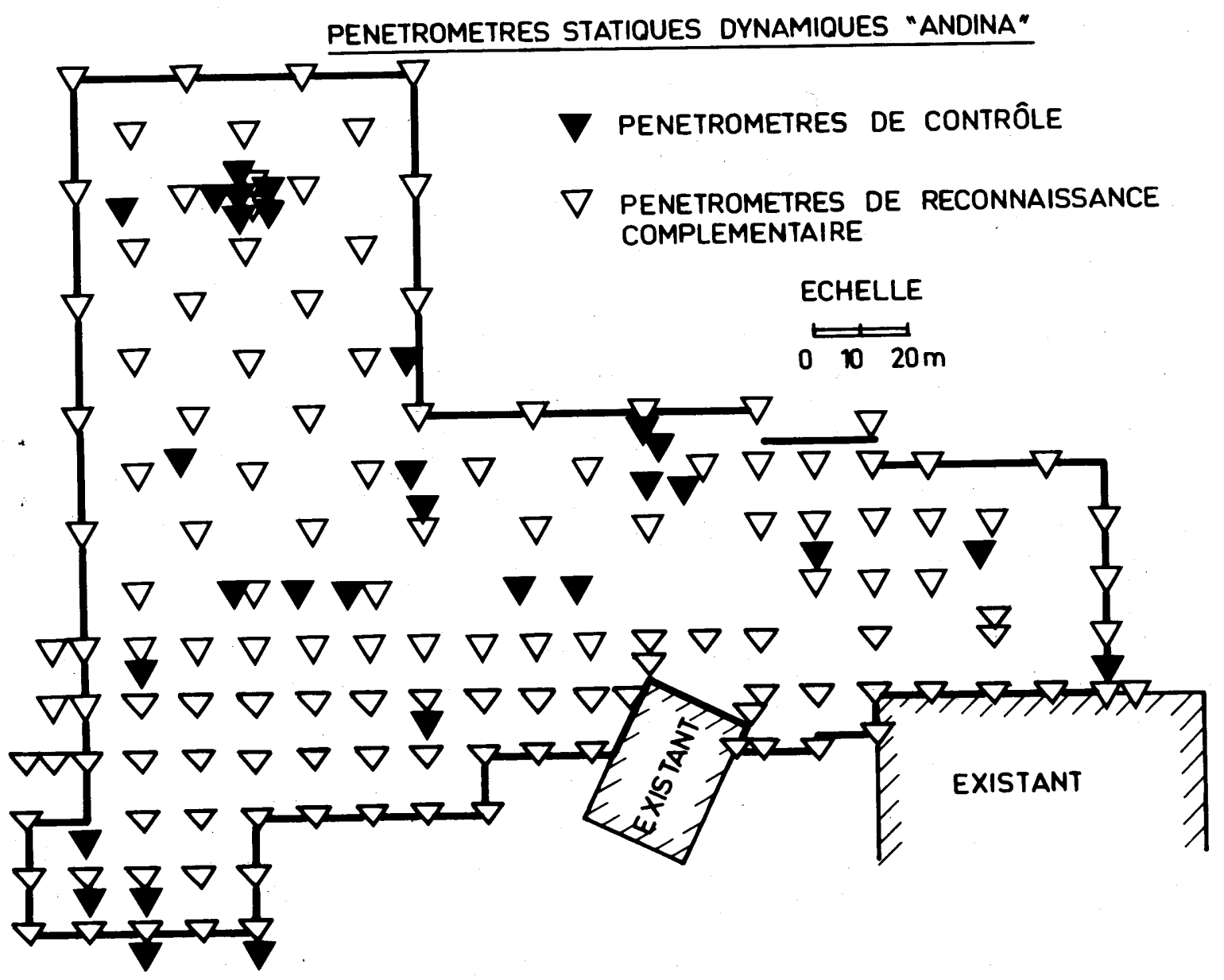


Détail du traitement de dallage

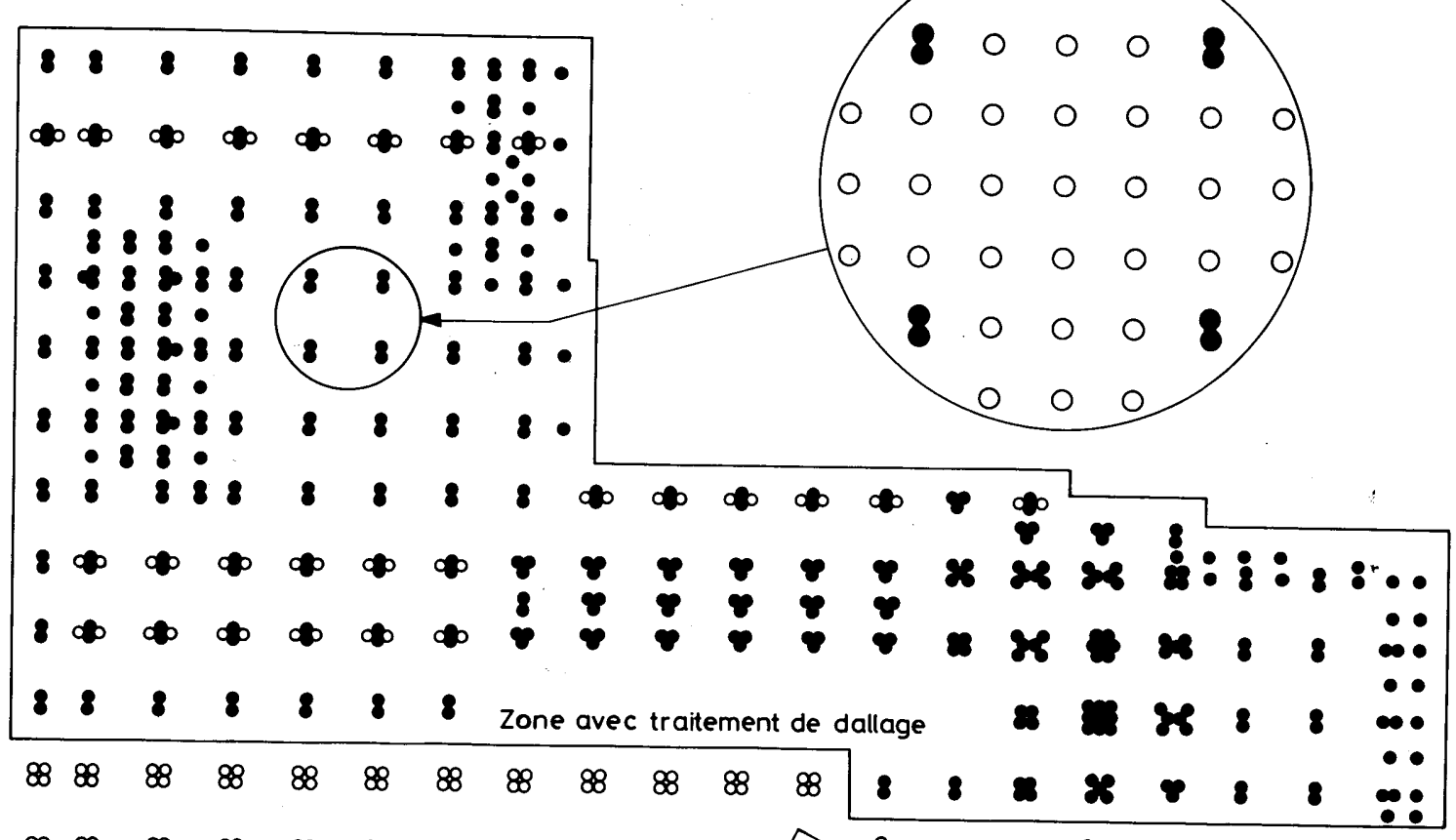

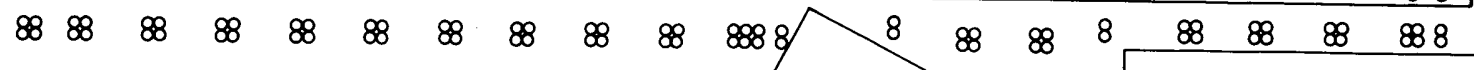
$\& \& \& 88$

$\$$

$\begin{array}{cccccc}8 & 8 & 8 & 8 & 88\end{array}$

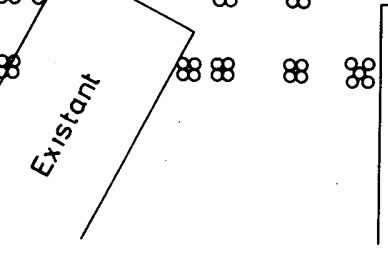

- Colonnes ballastées cimentées en tête O Colonnes ballastées ordinaires
Existant

Fig. 3

Atelier pour réalisation de colonnes ballastées, en action
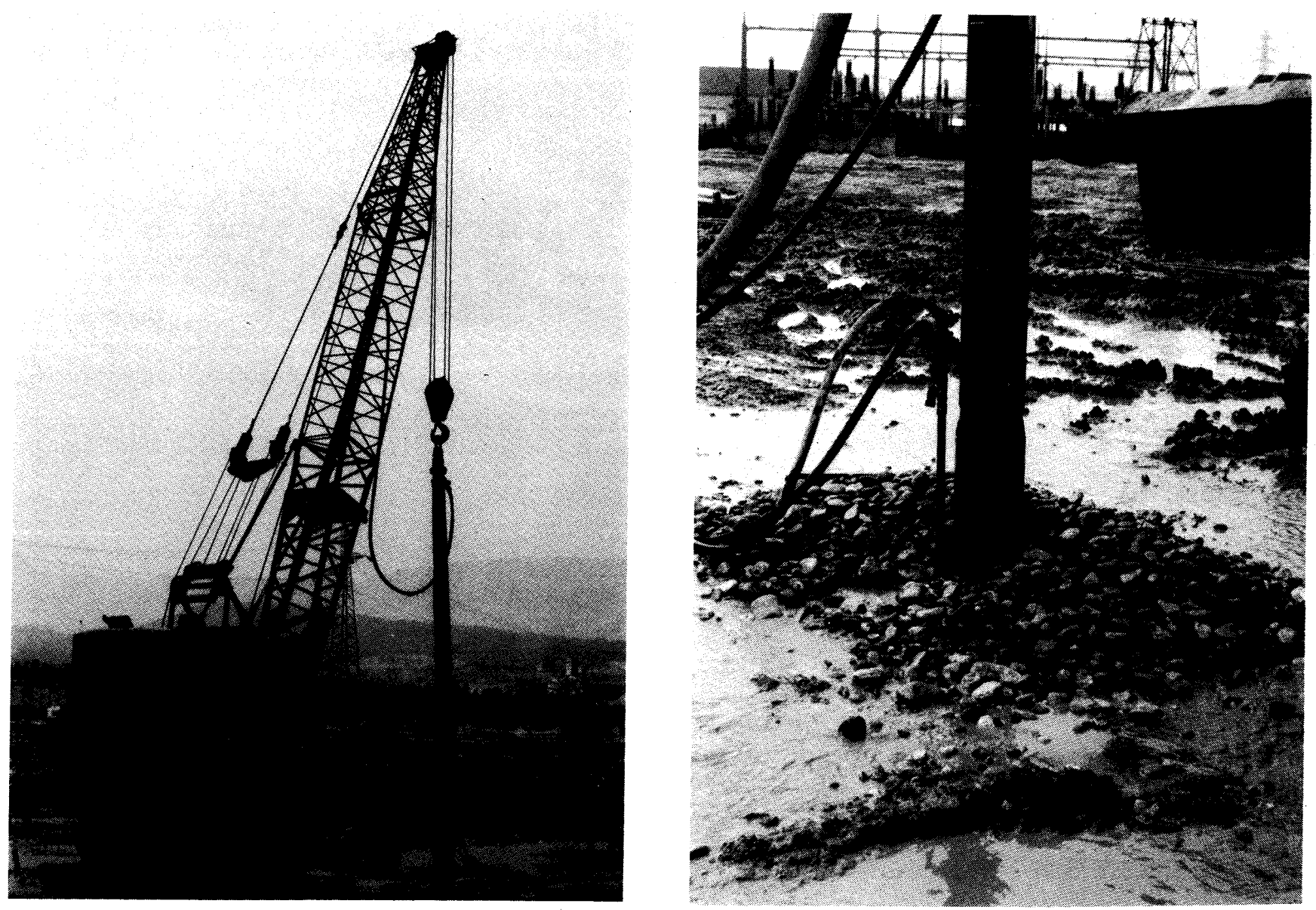
plus serré. II en résulte un cortège d'améliorations pour l'utilisateur: augmentation de l'angle de frottement donc de la résistance au cisaillement, diminution de la compressibilité, diminution de la perméabilité, diminution de la sensibilité aux séismes par l'effet d'écrouissage dynamique. Le traitement est effectué par points, dont la profondeur et la maille sont adaptées aux conditions du projet. La densité de la maille de traitement est en général choisie en fonction du rayon d'action du vibreur de telle sorte que le résultat du traitement soit homogène. Les gains de portance et la diminution de tassements pour les fondations, l'augmentation de la sécurité au glissement pour les talus, la diminution de perméabilité pour les digues ou les fonds de fouilles, atteignent souvent des proportions spectaculaires, qu'attestent de nombreuses réalisations de par le monde.

\subsection{La colonne ballastée, son principe}

II en va autrement lorsque le sol comporte en partie ou en totalité des particules fines tels que limons et argiles. Les forces interparticulaires sont grandes vis-à-vis de la masse des particules lorsque celles-ci sont petites et ne peuvent être concurrencées par les forces résultant de l'accélération communiquée aux particules par la source vibratoire. Par ailleurs, ces mêmes sols fins, à faible perméabilité, ne peuvent expulser leur eau interstitielle que très lentement et sous chargement statique prolongé. II s'ensuit que dans les sols fins, et à degré de saturation élevé, il n'y a guère de modification de l'arrangement du squelette sous l'effet dynamique de la vibration.

La vibroflottation étant alors partiellement ou totalement inefficace (de même d'ailleurs que les procédés de compactage depuis la surface), on a recours à une incorporation de matériaux sélectionnés. Ces matériaux d'apport sont en général des graves propres. Ils sont introduits depuis la surface dans l'espace annulaire qui existe entre le vibreur et la paroi du trou que le vibreur a fait dans le terrain. Les matériaux incorporés cheminent, grâce à la vibration, jusqu'à la pointe du vibreur.

On remonte alors le vibreur en vibration, tout en lui imprimant des mouvements verticaux alternatifs à résultante ascendante, de manière à constituer progressivement, en partant du bas, une colonne de matériaux sélectionnés, intensément compactés, appelée conventionnellement "colonne ballastée ».

On notera le rôle multiple du vibreur: création du trou, compactage des couches naturelles compactables, maintien du forage pendant l'incorporation, compactage du matériau incorporé. De plus, le procédé est autorégulateur dans la mesure où, à chaque niveau, l'incorporation est d'autant plus importante que le sol à ce niveau est faible. II en résulte un effet très bénéfique d'homogénéisation du terrain.

De par sa forme une colonne ballastée ressemble à un pieu, mais ce n'est pas un pieu. II faut donc bannir l'appellation de "pieu-colonne-ballastée " qui favorise une conception erronnée de ce système de fondation.

Une colonne ballastée n'existe que grâce à la réaction du terrain environnant. Plus l'effet de confinement est important, plus la colonne est apte à supporter des charges verticales. Une surcharge en surface autour de la colonne augmentera donc la capacité de la colonne. C'est l'ensemble constitué par la colonne et le terrain environnant qui, jusqu'en tête, assure solidairement la reprise de la charge.

Le bâtiment fondé sur un terrain traité par colonnes ballastées repose, selon les cas, sur des semelles superficielles ou sur un radier.

Les colonnes sont, soit disposées en files ou en groupes selon que les appuis sont filants ou isolés, soit réparties sous un radier ou un dallage. Dans tous les cas la fondation repose à la fois sur les colonnes et sur le terrain entre les colonnes. Une disposition adéquate des colonnes et des armatures dans les semelles doit être prévue dans le cas de moments ou de charges inclinées.

\subsection{La philosophie des procédés}

de traitement de sol

par vibroflottation et colonnes ballastées

La définition des traitements de sol par ces procédés est conduite de telle sorte que l'on vérifie à la fois que la portance est suffisante et que les tassements (absolus et différentiels) sont admissibles.

Les traitements sont conçus, selon les cas, soit en dispositifs "flottants », soit en dispositifs «de transition» appuyés en pied sur une couche portante.

II serait vain de mettre en compétition les performances unitaires des pieux classiques et des colonnes ballastées. Mais il est à présent reconnu que les colonnes ballastées ne sont pas les «pieux du pauvre » et qu'elles ressortissent bien au contraire à la catégorie des procédés qui, au même titre que, par exemple, la fameuse "terre armée.», font participer fonctionnellement la masse du sol et en exploitent les ressources négligées par les procédés traditionnels.

II ne s'agit pas d'une invention récente, loin s'en faut. Monsieur J. Kerisel nous a aimablement signalé une référence historique de 1830 , citée dans un numéro du "Mémorial de l'Officier du Génie " de 1832, par le capitaine M. Moreau. Cet auteur y expose une fondation expérimentale sous forme de "pilots de sable " réalisés à l'Arsenal d'Artillerie de Bayonne dans des terrains vaseux, et qui avaient donné toute satisfaction.

Pour le moment il n'existe pas, à notre connaissance du moins, de méthode rationnelle de dimensionnement des colonnes ballastées, ce qui est considéré, dans notre pays du moins, comme un lourd handicap. II semble que le pragmatisme éclairé de nos prédécesseurs de 1830 n'a pas été intégralement transmis à notre génération.

On notera au passage que les projeteurs appelés à calculer des portances de pieux classiques ne sont guère mieux armés en matière de méthode théorique rationnelle, sans que cela entrave particulièrement les applications de cette technique.

\section{Les essais de chargement de colonnes ballastées exécutés à l'occasion du chantier de Chamnord}

\subsection{Description des terrains}

Les essais ont été réalisés dans la Zone II décrite au paragraphe 1.1 .

Le terrain est constitué de $4 \mathrm{~m}$ de limons reposant sur des couches alternées de puissance métrique de graves, tantôt propres, tantôt argileuses. La nappe phréatique, à l'endroit et au moment des essais, est à $1,50 \mathrm{~m}$.

Ce faciès peut être schématisé par un bicouche mou/dur. La reconnaissance précise des terrains à l'endroit des essais a été faite par carottage intégral en vibropercussion, pressiomètre Ménard, et pénétromètre statique Andina, avec essais de laboratoire. Les principales caractéristiques géotechniques observées sont données sur le tableau de la figure 4 .

\subsection{Description du dispositif d'essai}

Les deux colonnes ballastées essayées ont les caractéristiques suivantes :

Colonne ballastée standard: traversant les limons et ancrée de 0,50 m dans les graves,

Colonne ballastée cimentée : ballast cimenté au coulis dosé à $325 \mathrm{~kg}$ de ciment CLK, sur la traversée des limons, et colonne ballastée standard de $4,50 \mathrm{~m}$ de profondeur sous le toit des graves.

Le diamètre apparent des colonnes est d'environ $1 \mathrm{~m}$ pour les colonnes standard et $0.80 \mathrm{~m}$ pour les parties cimentées.

Chaque colonne est chargée en tête, après recépage sur 0,60 m, par l'intermédiaire d'une semelle en béton armé de 


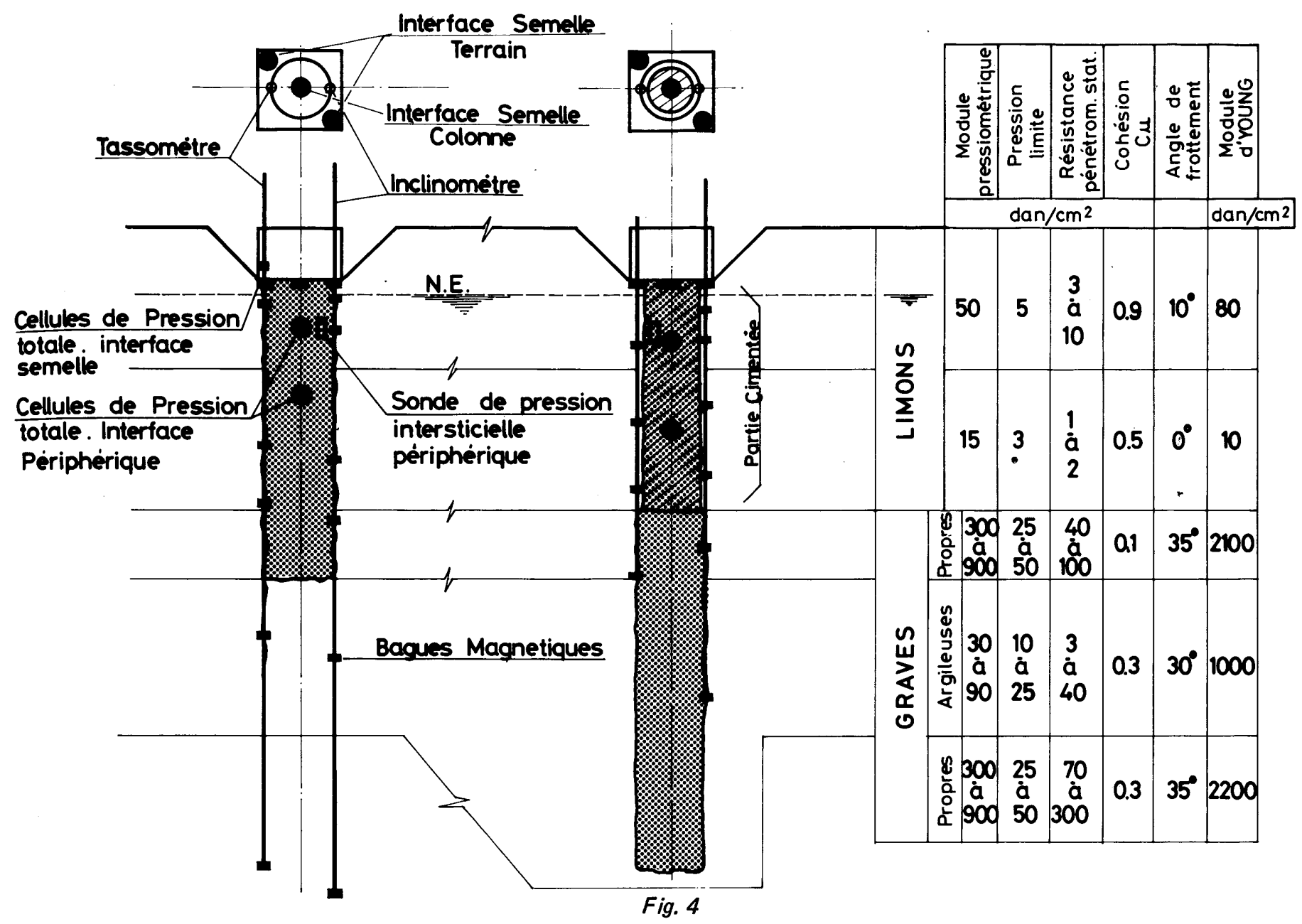

Fig. 4 bis Dispositif de chargement

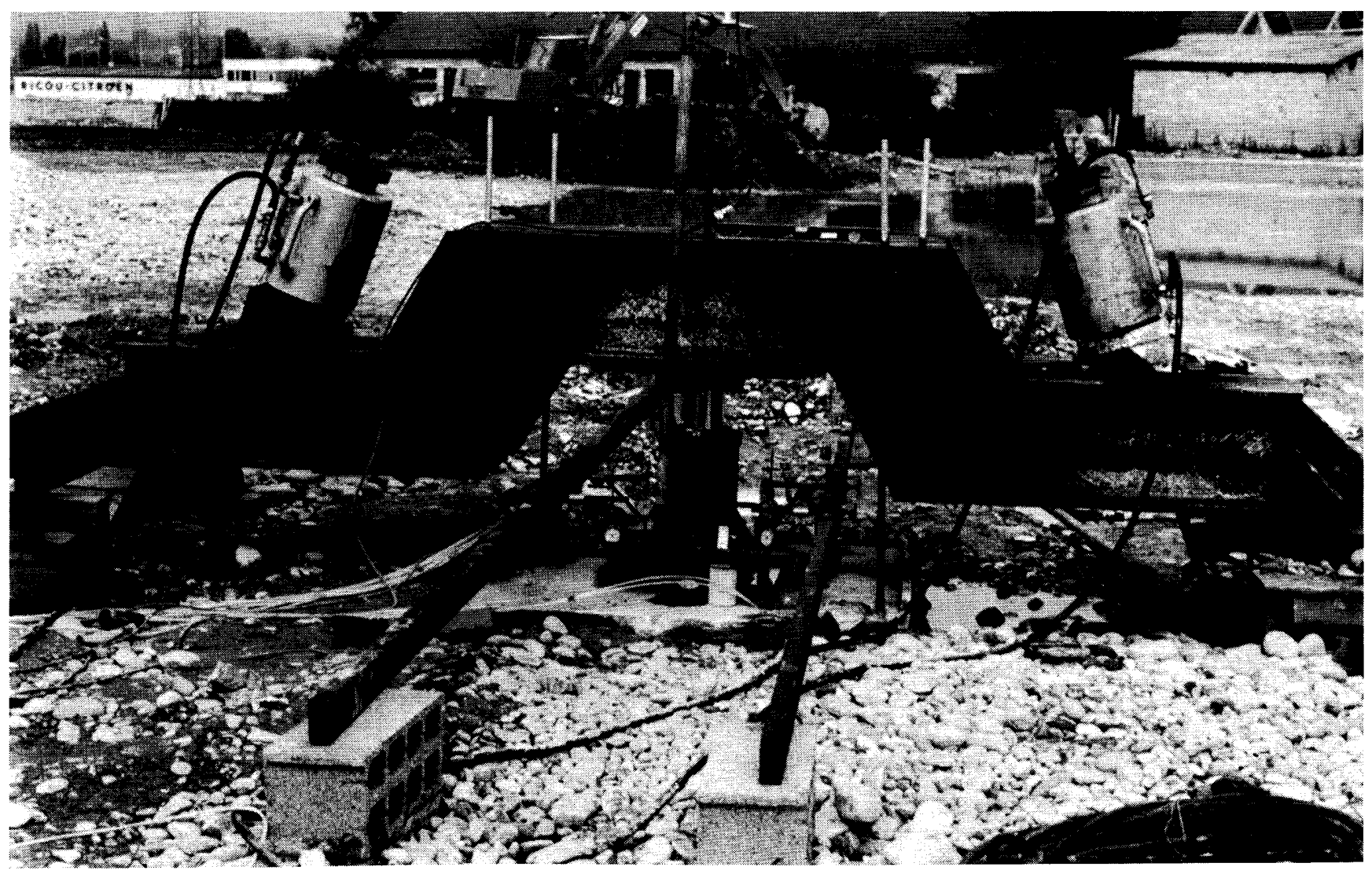


$1,20 \times 1,20 \times 0,60 \mathrm{~m}$. Cette semelle est surmontée d'une poutre métallique horizontale, avec une rotule et un dynamomètre intercalés. La poutre est chargée à ses extrémités par 2 vérins hydrauliques couplés prenant réaction sur des tirants scellés au terrain (fig. 4 bis). Le dispositif permet de développer $1 \mathrm{MN}$.

L'appareillage d'auscultation permet d'enregistrer la courbe effort-tassement de' la semelle (fig. 5), et de mesurer la pression totale, la pression interstitielle, les déplacements selon l'axe et le rayon, en divers points au contour de la colonne.

Les contraintes totales sont mesurées par des cellules Glotzl. En particulier, la distribution des contraintes à la sous-face de la semelle est observée par trois de ces cellules.

Aucun appareillage d'auscultation ne peut valablement être placé à l'intérieur de la colonne. Toutes les mesures sont faites au contour. Les déplacements notamment sont mesurés par tassomètres et inclinomètres verticaux quasi tangents à la colonne.

\subsection{Programme de chargement}

Colonne ballastée standard :

Cycle $1: 240 \mathrm{kN}$ (24 tonnes)

incréments de $80 \mathrm{kN}$, paliers d'une heure.
Cycle 2 : $400 \mathrm{kN}$ (40 tonnes) montée à $240 \mathrm{kN}$ puis incréments de $80 \mathrm{kN}$, paliers d'une heure.

Cycle $3: 480 \mathrm{kN}$ (48 tonnes) montée incrémentale et paliers courts jusqu'à $400 \mathrm{kN}$.

palier de 20 heures à $480 \mathrm{kN}$

Cycle 4 : $1000 \mathrm{kN}$ (100 tonnes)

palier d'1 heure à

palier $d^{\prime} 1$ heure à

$720 \mathrm{kN}$

$800 \mathrm{kN}$

palier de 0,5 heure à $880 \mathrm{kN}$

palier de 0,25 heure à $960 \mathrm{kN}$

palier de 0,33 heure à $1000 \mathrm{kN}$

Colonne ballastée cimentée :

Cycle $1: 400 \mathrm{kN}$ (40 tonnes) incréments de $80 \mathrm{kN}$, paliers de 0,25 heure

Cycle 2: $1000 \mathrm{kN}$ (100 tonnes) palier de 3,25 heures à $480 \mathrm{kN}$ palier de 1,5 heure à $560 \mathrm{kN}$ palier de 2 heures à $640 \mathrm{kN}$ palier de 14 heures à $720 \mathrm{kN}$ palier de 1,5 heure à $800 \mathrm{kN}$ palier de 1,5 heure à $880 \mathrm{kN}$ palier d'1 heure à $960 \mathrm{kN}$ palier de 0,5 heure à $1000 \mathrm{kN}$

Fig. 5 Diagrammes effort-tassement
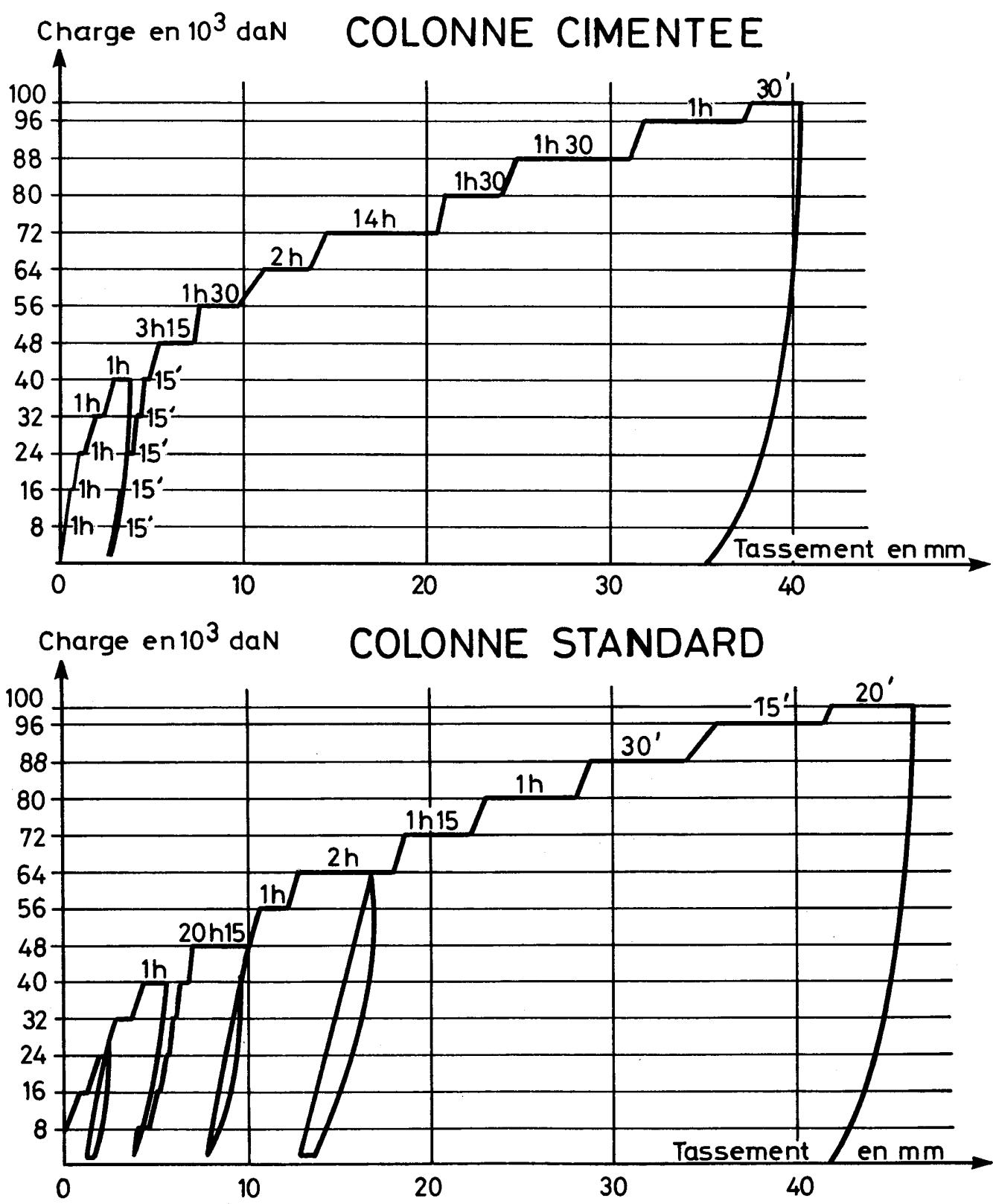


\subsection{Résultats}

\subsubsection{Courbe effort-tassement, courbe de fluage,} charge critique de fluage.

Les chemins de sollicitation suivis ne sont pas exactement identiques pour chacune des deux colonnes, mais la comparaison reste significative.

Sur les courbes enveloppes des diagrammes efforttassement on peut relever les caractéristiques suivantes :

\begin{tabular}{c|c|c}
\hline \multirow{2}{*}{ effort } & \multicolumn{2}{|c}{$\begin{array}{c}\text { tassements } \\
\mathrm{mm}\end{array}$} \\
\cline { 2 - 3 } en kN & colonne standard & colonne cimentée \\
\hline & & \\
240 & 2 & 1 \\
400 & 5 & 3 \\
560 & 11 & 8 \\
720 & 42 & 15 \\
1000 & & 38 \\
& & \\
\hline
\end{tabular}

Les diagrammes de fluage (fig. 6 et 7) font apparaître le tassement de la tête de colonne, pour une charge donnée constante, en fonction du logarithme du temps. Lorsque la courbe est une droite, il y a stabilisation du tassement selon une loi logarithmique. C'est le cas pour les faibles charges. Quand la charge augmente, la pente de la droite augmente ce qui indique une vitesse de tassement plus grande. Enfin pour des charges élevées, la courbe n'est plus droite, mais concave vers le haut ce qui indique qu'il n'y a plus tendance à la stabilisation mais mise en plasticité et grands déplacements dans une partie importante de la fondation, c'est-à-dire rupture.

La charge critique, qui marque la frontière entre le domaine stable (pseudo-élastique) et le domaine instable (plastique), apparaît bien sur le graphique où sont portées les pentes des courbes de fluage en fonction de la charge appliquée.

Les deux colonnes ont manifesté une charge critique de $500 \mathrm{kN}$ (50 tonnes) environ. La vitesse de fluage est 20 à $25 \%$ plus faible pour la colonne cimentée.

\subsubsection{Fluage en palier de longue durée (fig. 8)}

Sur la colonne ballastée standard, soumise à $480 \mathrm{kN}$ pendant 20 heures, on note $1 \mathrm{~mm} /$ décade entre 0 et 6 heures puis stabilisation (tassement nul). On est pourtant très près de la charge critique.

Sur la colonne ballastée cimentée, le palier de 14 heures à $720 \mathrm{kN}$, soit 1,5 fois la charge critique, montre qu'il n'y a pas tendance à la stabilisation : $1,4 \mathrm{~mm} /$ décade entre 0 et 0,5 heures, $2,6 \mathrm{~mm} /$ décade entre 0,5 et 8 heures, $3,8 \mathrm{~mm} /$ décade entre 8 et 13 heures.

Fig. 8
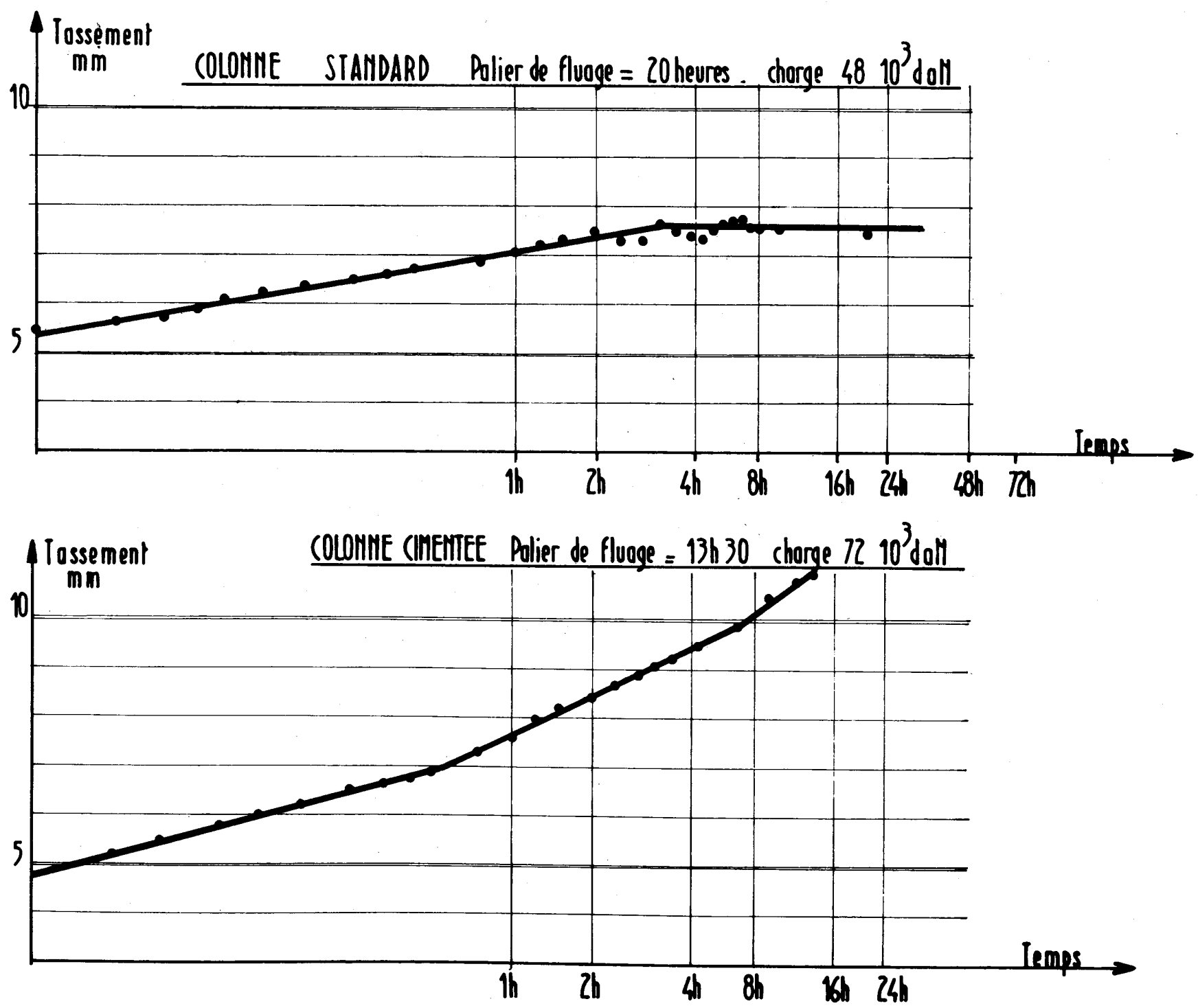

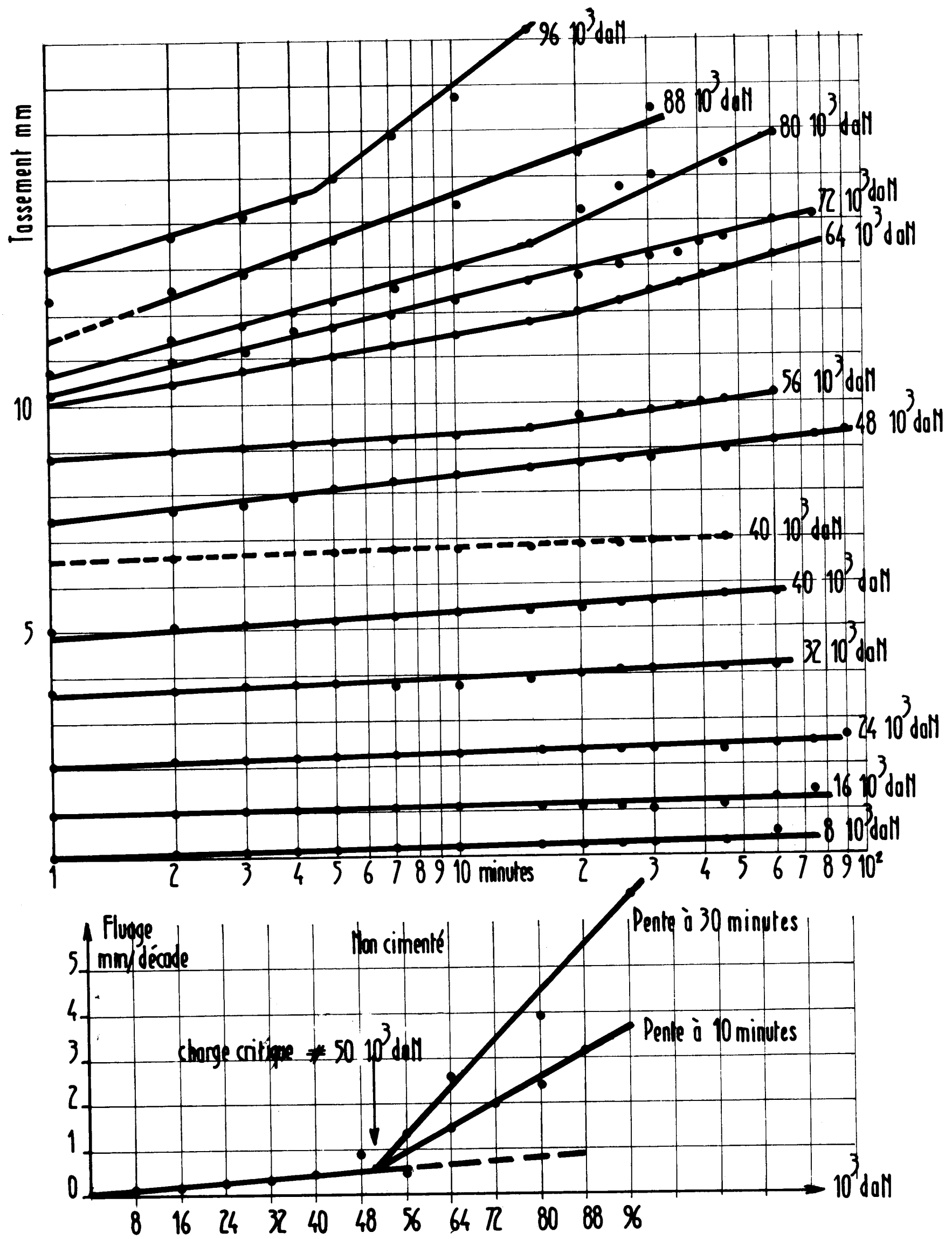

Fig. 6 Colonne standard - Diagrammes de fluage à effort constant - Détermination de la charge critique de fluage 

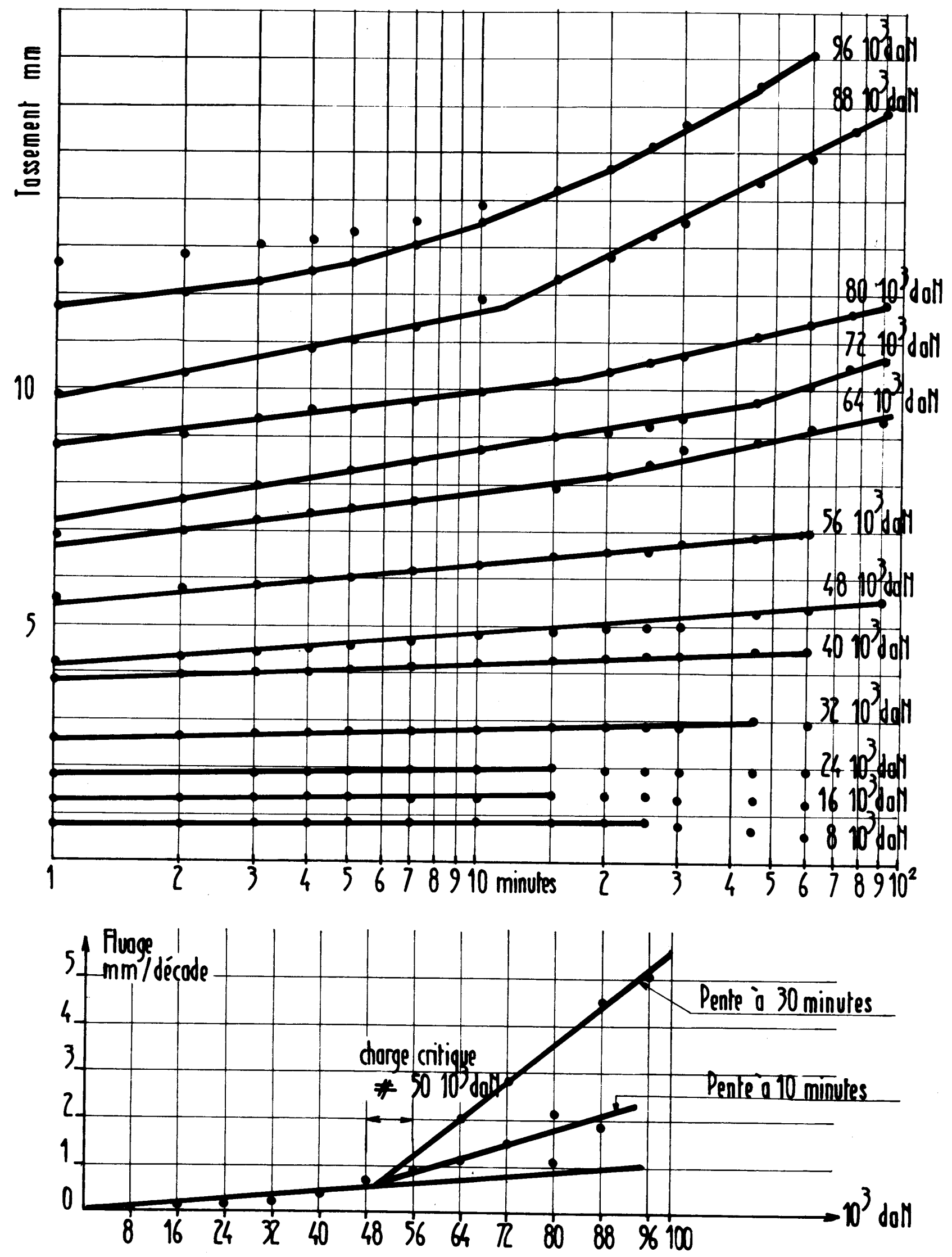

Fig. 7 Colonne cimentée - Diagrammes de fluage à effort constant - Détermination de la charge critique de fluage 


\section{COLONNE STANDARD}

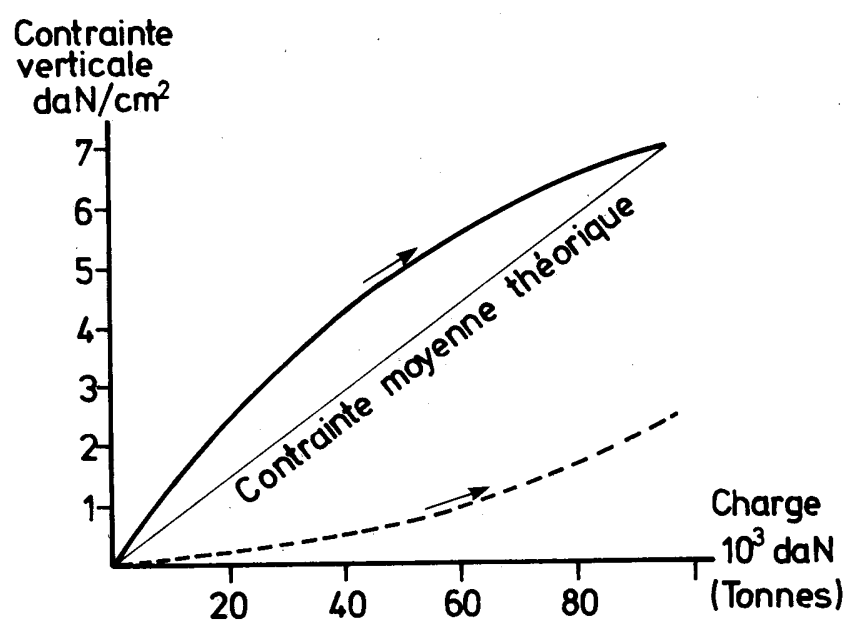

\section{COLONNE CIMENTEE}

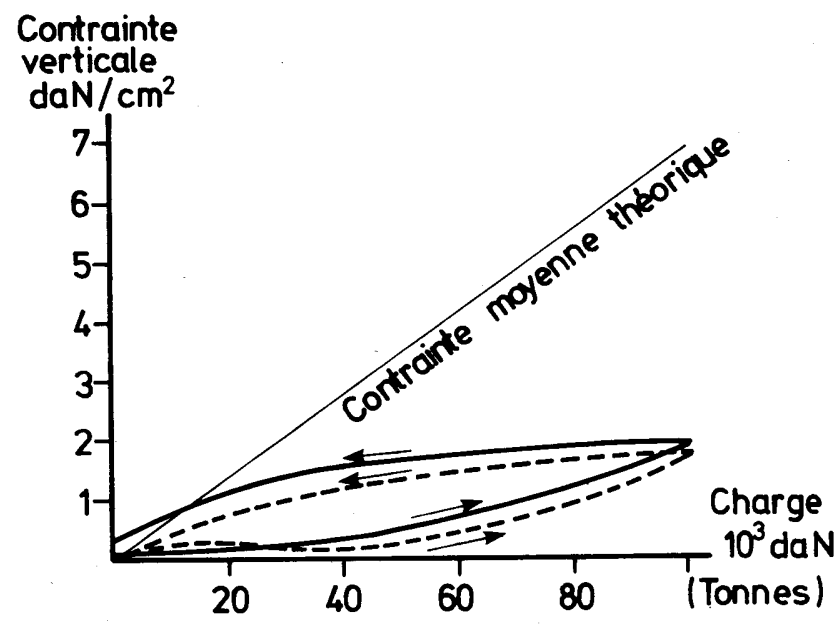

\section{CONTRAINTE MESUREE ( 1 cellule) SUR COLONNE CONTRAINTE MESUREE (moyenne 2 cellules) SUR SOL}

Fig.9

\subsubsection{Distribution des contraintes sous la semelle (fig. 9)}

On ne peut accorder aux résultats des mesures effectuées qu'une valeur qualitative, la valeur absolue des contraintes mesurées étant certainement inexacte puisqu'elle ne permet pas de recalculer la charge totale en faisant le produit par la surface d'application.

Encore les résultats sont-ils paradoxaux sur le seul plan qualitatif puisque le contraste entre contrainte sur colonne et contrainte sur sol est moins important sur la colonne cimentée que sur la colonne standard.

En grossière approximation on peut admettre que, dans les conditions de l'essai, la contrainte sur la colonne est égale à 1,5 à 4 fois la contrainte sur le sol.

3.4.4. Tassomètre (fig. 10)

On observe un comportement relativement identique sur les deux colonnes d'essai, caractérisé par une disparition des déformations axiales à partir de $2 \mathrm{~m}$ de profondeur environ.
3.4.5 Inclinomètre, contrainte radiale, pression interstitielle

Le résultat de ces mesures est quasi nul ou ininterprétable.

\subsection{Conclusions}

Les performances de la colonne standard sont très intéressantes sur le plan de la force portante, la charge critique étant d'environ $500 \mathrm{kN}$ (50 tonnes). Celles de la colonne cimentée sont moins brillantes puisque sa charge critique n'est pas supérieure. Toutefois la colonne cimentée apporte un gain significatif sur le plan du tassement, celui-ci étant inférieur de $50 \%$ pour une charge égale à la moitié de la charge critique, et inférieur de $30 \%$ pour une charge égale à la charge critique.

Le siège des déformations des colonnes sous charge se situe essentiellement dans les deux premiers mètres en tête.

On notera que la mise en charge de la colonne cimentée a été faite 40 jours après sa confection, le coulis n'ayant

Fig. 10

\section{Tassométres Magnétiques S.I.F.}

\section{Colome Standard}

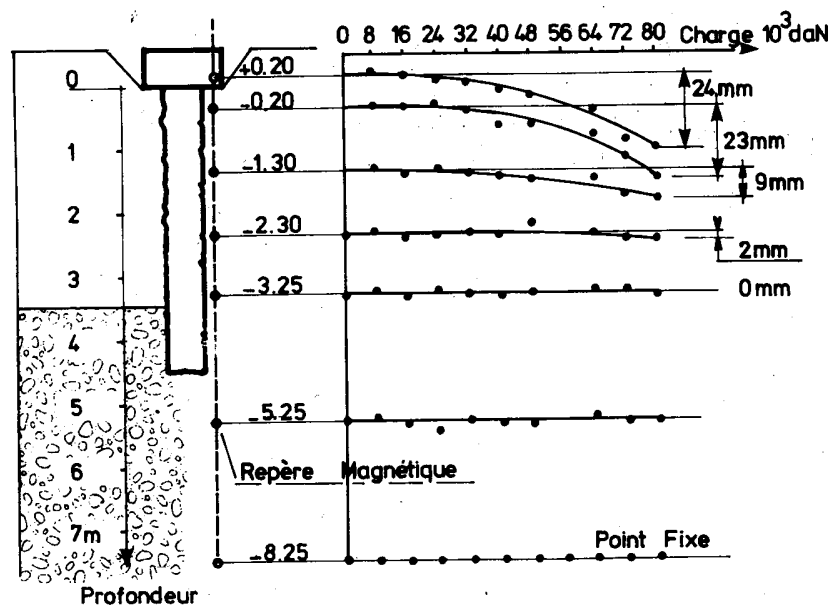

\section{Colonne Cimentèe}

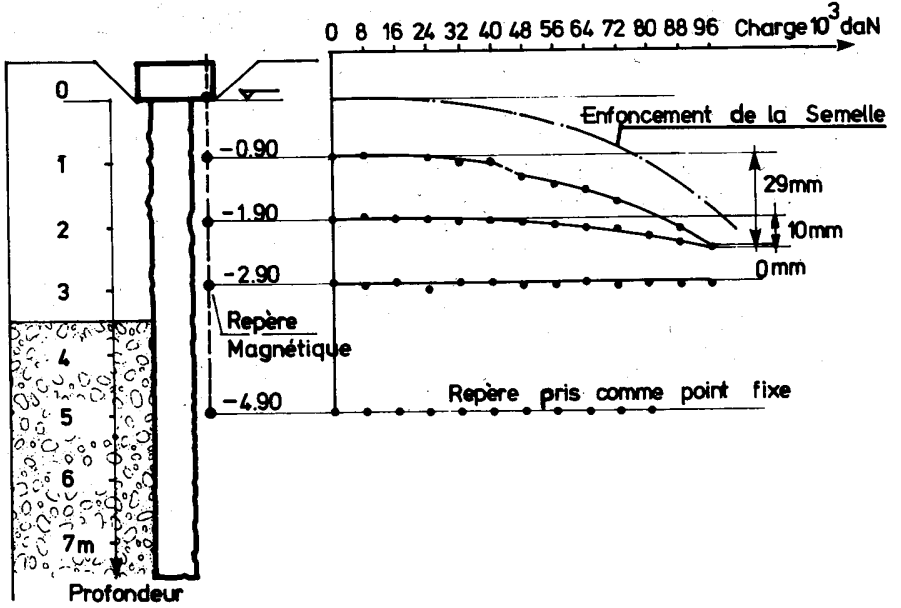




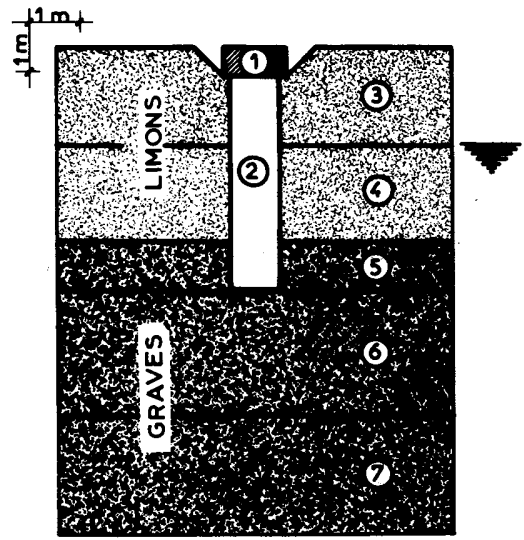

\begin{tabular}{|c|c|c|c|c|}
\hline & $\begin{array}{c}\varphi \\
\text { degres }\end{array}$ & $\begin{array}{c}C \\
\text { daN/cm }\end{array}$ & $\begin{array}{c}E \\
\text { daN } / \mathrm{cm}^{2}\end{array}$ & $\nu$ \\
\hline (1) & 0 & 1000 & 280000 & 0.25 \\
\hline (2) & $40^{\circ}$ & 0.5 & 3300 & 0.20 \\
\hline (3) & $10^{\circ}$ & 0.9 & 80 & 0.33 \\
\hline (6) & 0 & 0.5 & 10 & 0.49 \\
\hline (5) & $35^{\circ}$ & 0.1 & 2100 & 0.20 \\
\hline (2) & $30^{\circ}$ & 0.3 & 1000 & 0.20 \\
\hline (D) & $35^{\circ}$ & 0.1 & 2200 & 0.20 \\
\hline
\end{tabular}

Fig. 11 Colonne standard - Calcul par la méthode des éléments finis - Caractéristiques rhéologiques

donc, au moment de l'essai, qu'une résistance en compression simple sur éprouvettes de l'ordre de $1,2 \mathrm{MPa}$, la résistance finale à long terme du même coulis se situant autour de $2 \mathrm{MPa}$.

On notera également que le mode d'exécution nécessité par la mise en place du coulis dans la partie traitée ne permet pas d'obtenir un bourrage aussi énergique du ballast dans la partie cimentée que sur une partie traitée de facon standard. II en résulte d'ailleurs une section de colonne cimentée inférieure de $35 \%$ environ à la section d'une colonne standard. Le gain apporté par la cohésion due à la cimentation est donc en partie neutralisé par la diminution de la section.

En supposant que la contrainte sous la semelle se répartisse entre colonne et sol autour de la colonne selon le rapport suivant :

$\frac{\text { contrainte sur colonne }}{\text { contrainte sur le sol }}=3$

on calcule que pour une charge de $500 \mathrm{kN}$ (50 tonnes) la contrainte sur la colonne standard est de 0,5 $\mathrm{MPa}$ et sur la colonne cimentée de 0,62 $\mathrm{MPa}$.

On admettra, en première approximation, que le gain apporté par la cohésion sur le plan de la force portante apparaît dans cet écart entre taux de travail. En tablant sur une résistance du coulis de ciment de $2 \mathrm{MPa}$ à long terme contre $0,2 \mathrm{MPa}$ au moment de l'essai, on peut calculer par proportionnalité que la force portante à long terme pourrait être de l'ordre de $15 \%$ supérieure à celle observée à 40 jours.

Compte tenu d'un rapport de prix entre colonne ballastée cimentée et colonne standard qui, selon les sujétions d'exécution, est de l'ordre de deux ou plus, un intérêt économique très sensible vient s'ajouter aux performances mécaniques avantageuses de la colonne ballastée standard.

Dans la mesure du possible, et sauf contre-indication spécifique, on aurait avantage à rechercher des solutions faisant appel à la technique de base des colonnes ballastées, quitte à multiplier les colonnes standard, l'effet de groupe apportant d'ailleurs un accroissement du coefficient de sécurité réel.

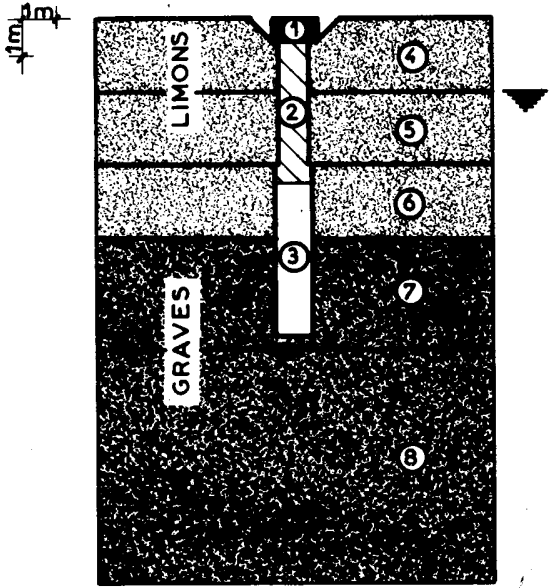

\begin{tabular}{|c|c|c|c|c|}
\hline & $\begin{array}{c}\varphi \\
\text { degres }\end{array}$ & $\begin{array}{c}C \\
\text { doN } / \mathrm{mm}^{2}\end{array}$ & $\begin{array}{c}E \\
\text { doN } / \mathrm{cm}^{2}\end{array}$ & $\nu$ \\
\hline (1) & 0 & 1000 & 280000 & 0.25 \\
\hline$(2)$ & $40^{\circ}$ & 25 & 4500 & 0.20 \\
\hline (3) & $40^{\circ}$ & 0.5 & 3300 & 0.20 \\
\hline (4) & $10^{\circ}$ & 0.9 & 80 & 0.33 \\
\hline (5) & 0 & 0.5 & 10 & 0.49 \\
\hline (6) & $35^{\circ}$ & 0.1 & 2100 & 0.20 \\
\hline (7) & $30^{\circ}$ & 0.3 & 1000 & 0.20 \\
\hline (3) & $35^{\circ}$ & 0.1 & 2200 & 0.20 \\
\hline
\end{tabular}

Fig. 18 Colonne cimentée - Calcul par la méthode des éléments finis - Caractéristiques rhéologiques

\section{Résolution théorique par la méthode des éléments finis}

\subsection{Essais de laboratoire et hypothèses rhéologiques}

Ces essais ont été réalisés afin de déterminer les caractéristiques à prendre en compte dans les calculs.

Des essais triaxiaux consolidés à $50 \mathrm{kPa}$ non drainés réalisés sur le limon ont montré que la couche hors nappe était plus raide et plus cohérente que la couche sous la nappe $(E=8 \mathrm{MPa}$ et $\mathrm{Cu}=90 \mathrm{kPa}$ contre $\mathrm{E}=1 \mathrm{MPa}$ et $\mathrm{Cu}=50 \mathrm{kPa})$.

Les hypothèses de calcul sont récapitulées sur les figures 11 et 18 . II a été tenu compte de l'effet du vibreur sur les graves en place en retenant des valeurs de $E$ intermédiaires entre celles mesurées en laboratoire pour les densités mini et maxi des matériaux (norme ASTM).

Pour les matériaux des colonnes, dont les plus gros éléments atteignent $10 \mathrm{~cm}$, des essais ont été réalisés dans un œdomètre spécial $\varnothing 0,60 \times 0,50 \mathrm{~m}$, ainsi que des essais sur matériau homothétique de rapport 10 dans un moule $\varnothing 0,1.5 \mathrm{~m}$. Le module retenu est $E=330 \mathrm{MPa}$, correspondant à une masse volumique $\gamma \mathrm{d}=1,7 \mathrm{~g} / \mathrm{cm}^{3}$.

Les autres caractéristiques ont été déterminées de manière arbitraire. La cohésion choisie pour les colonnes cimentées, soit 25 daN $/ \mathrm{cm}^{2}$, est sensiblement supérieure à la valeur réelle probable, afin d'accentuer le contraste pour faire mieux ressortir le comportement spécifique de la colonne cimentée.

\subsection{Calculs par la méthode des éléments finis}

Ces calculs ont pour but la détermination du champ des contraintes et des déplacements dans les colonnes ballastées et à leur voísinage. Deux programmes ont été utilisés :

- un programme axisymétrique en élasticité

- un programme axisymétrique en élasto-plasticité, le critère d'écoulement étant le critère de Drucker. 

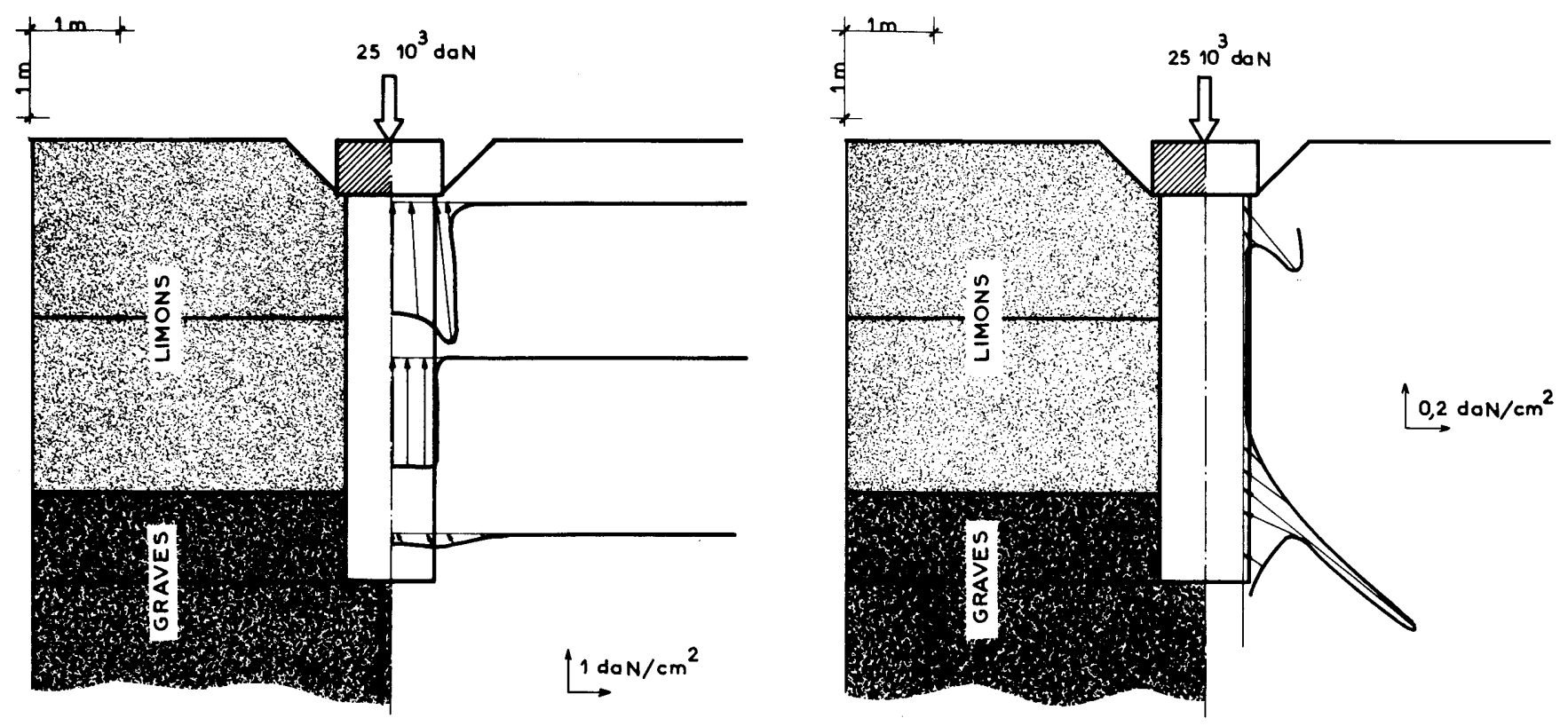

Fig. 12 Colonne standard - Calcul élastique - Accroissement de contrainte sur facettes horizontales
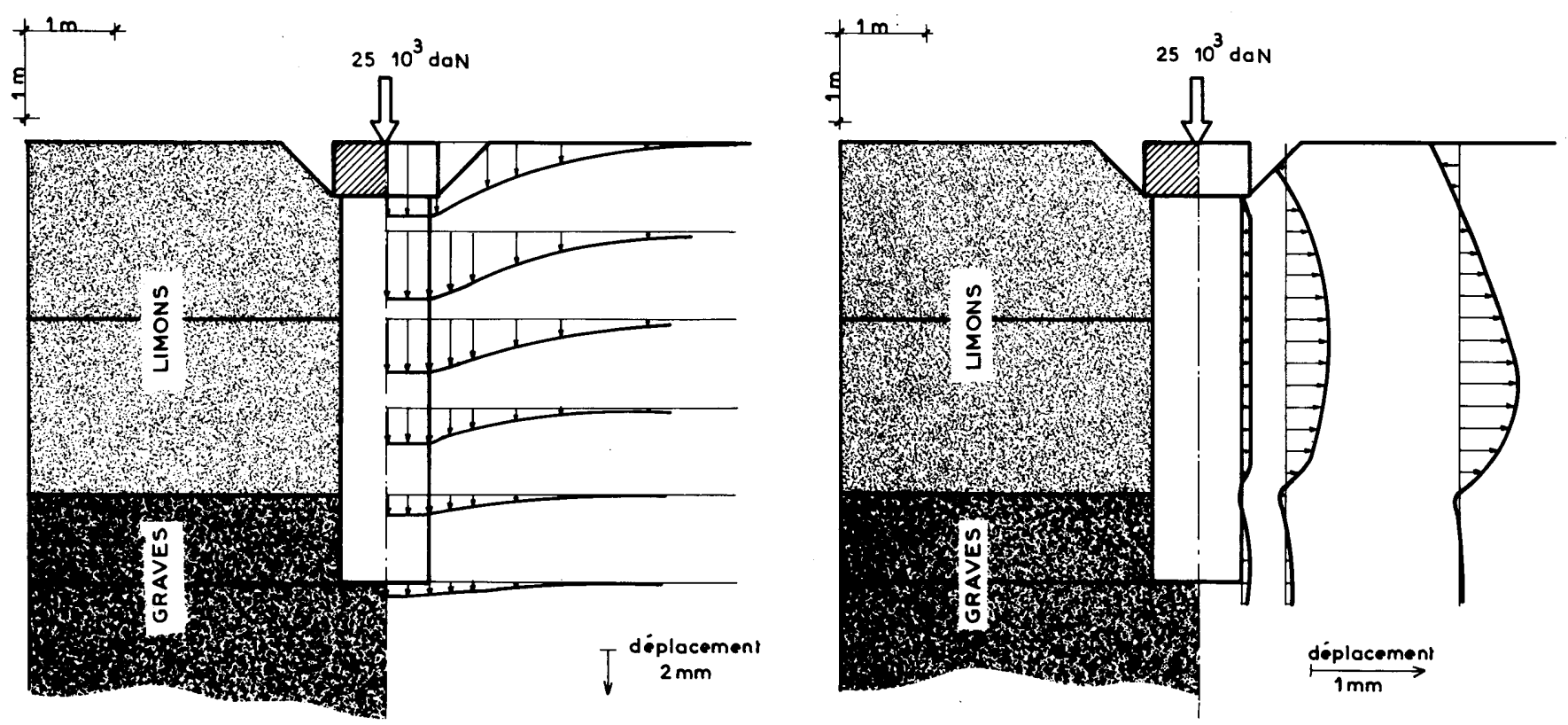

Fig. 14 Colonne standard - Calcul élastique - Déplacements verticaux (composante verticale) 


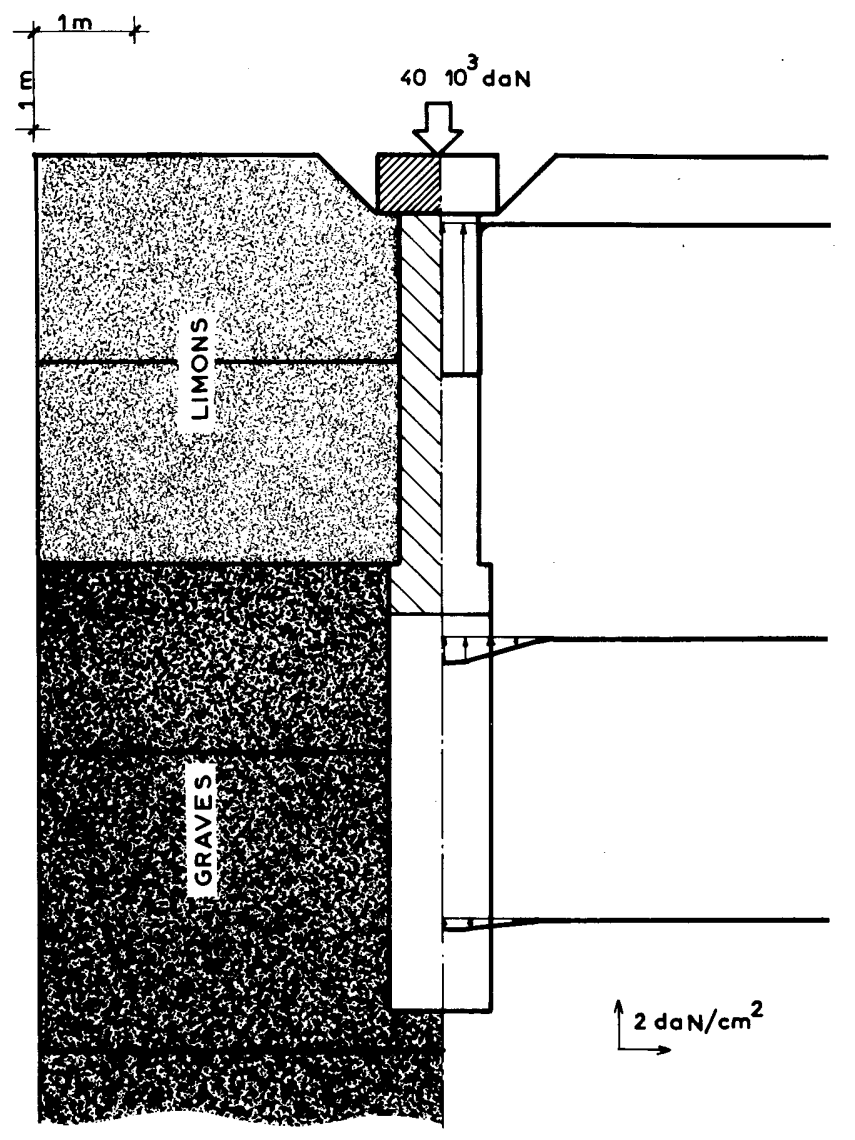

Fig. 19 Colonne cimentée - Calcul élastique - Accroissement de contrainte sur facettes horizontales

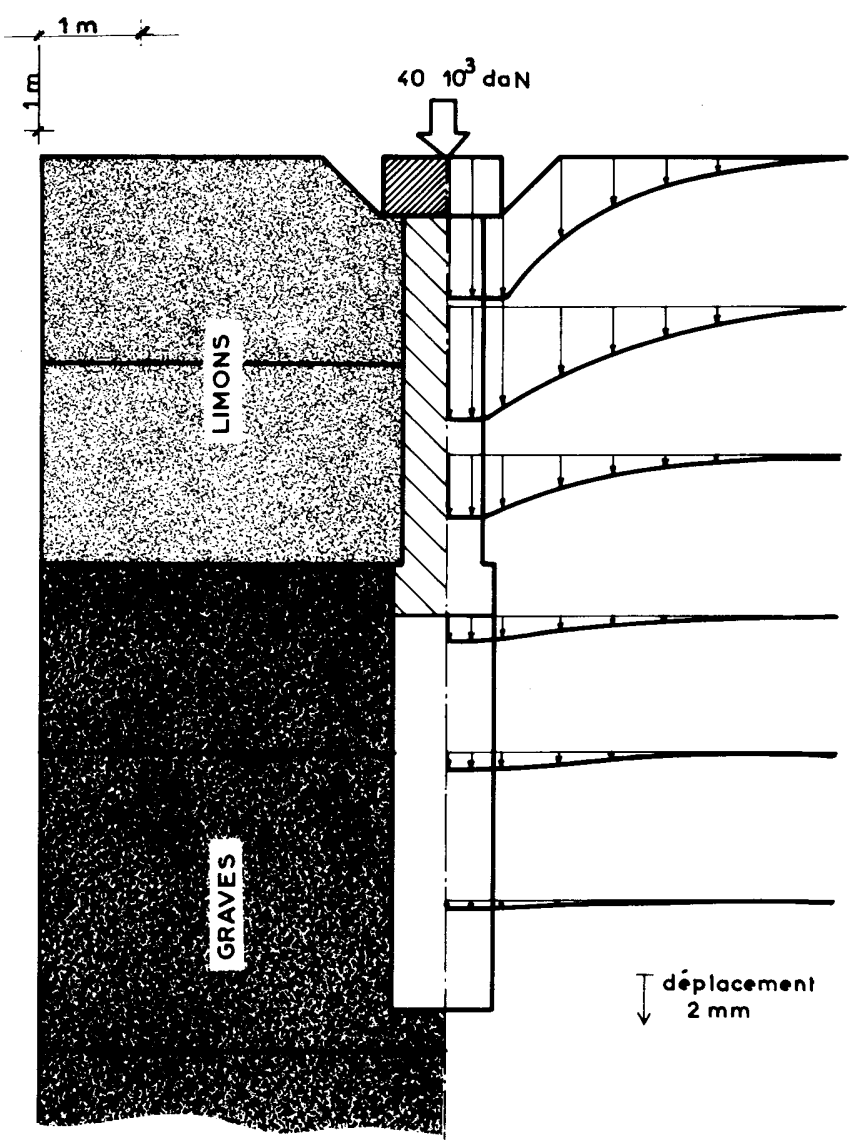

Fig. 21 Colonne cimentée - Calcul élastique - Déplace ments verticaux (composante verticale)

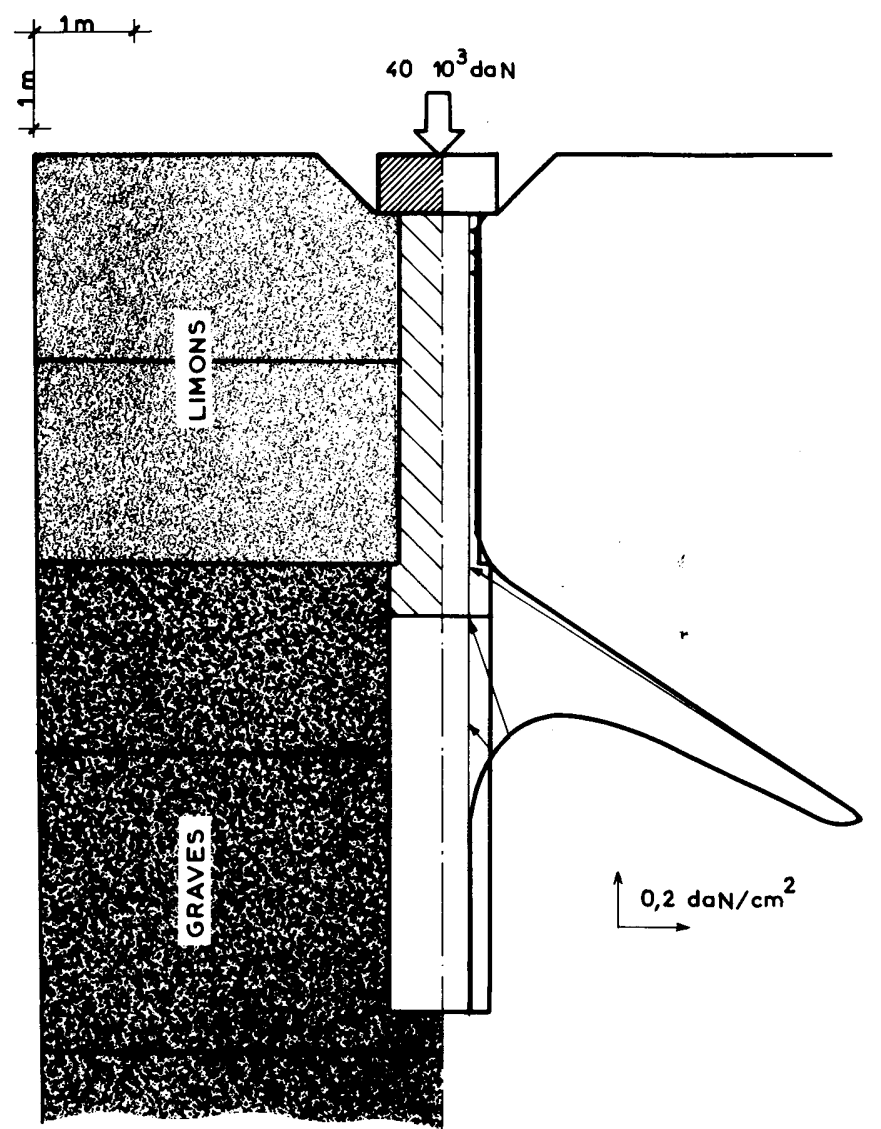

Fig. 20 Colonne cimentée - Calcul élastique - Accroissement de contrainte sur facettes verticales

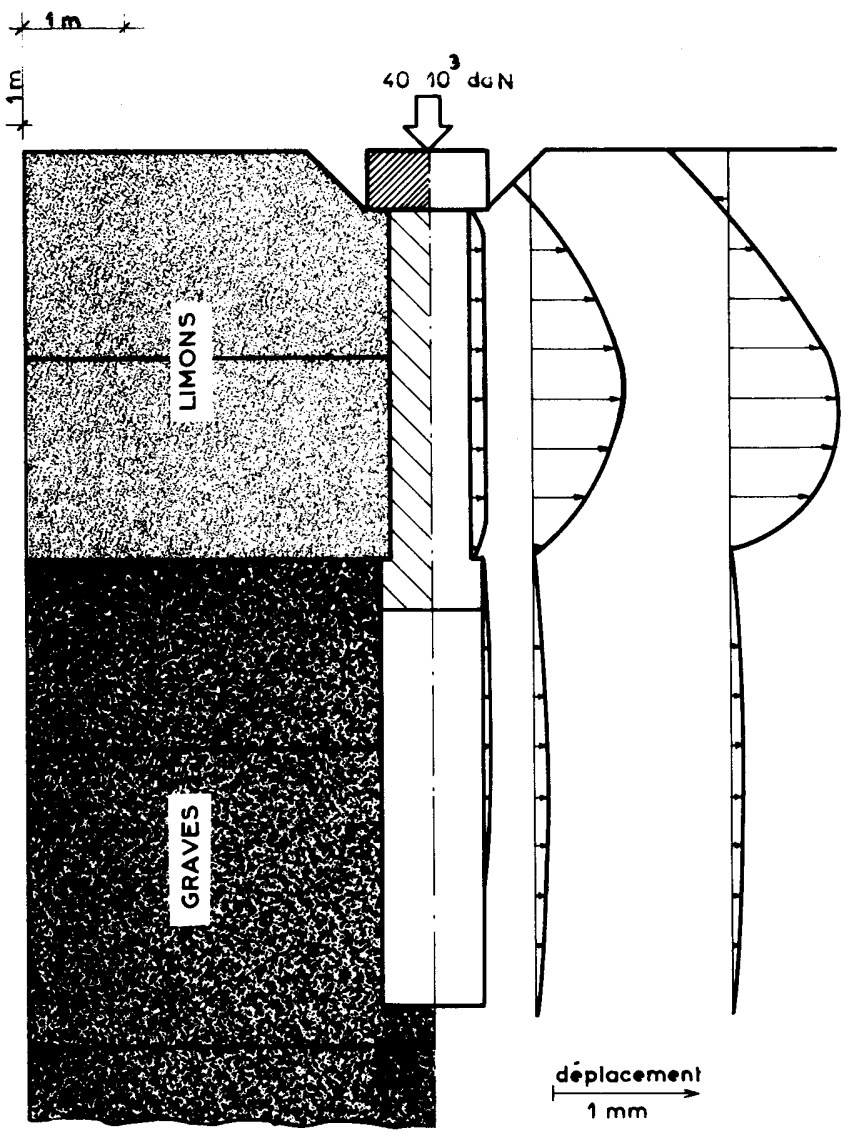

Fig. 22 Colonne cimentée - Calcul élastique - Déplacements horizontaux (composante horizontale) 


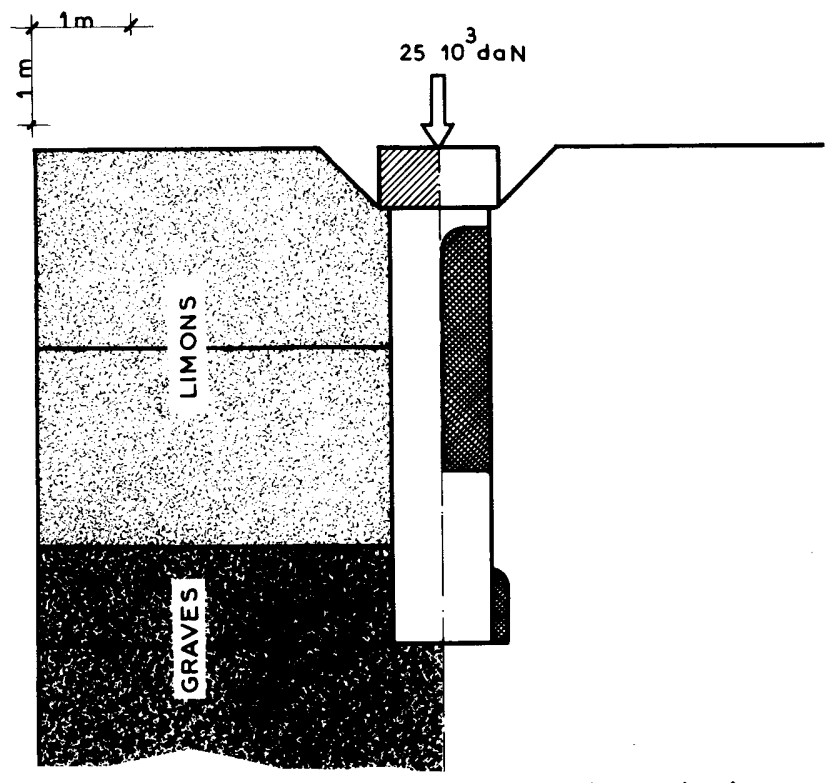

Fig. 16 Colonne standard - Calcul élasto-plastique Zones à l'état limite plastique sous $25.10^{3}$. daN

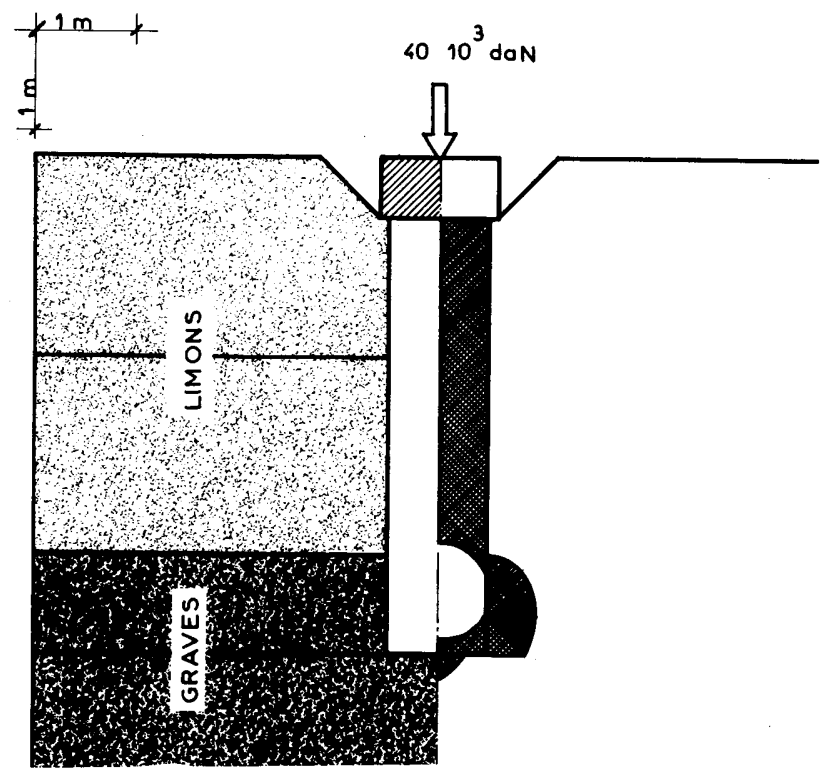

Fig. 17 Colonne standard - Calcul élasto-plastique Zones à l'état limite plastique sous $40.10^{3}$ daN

\subsubsection{Colonne ballastée standard chargée à $250 \mathrm{kN}$}

- Les contraintes sur les facettes horizontales sont concentrées dans la colonne et sont verticales sauf sous la tête et dans les graves (fig. 12). Le limon ne supporte pratiquement aucune contrainte, sauf en tête, sous la semelle. Dès que la colonne pénètre dans les graves, il y a un report non négligeable de contraintes sur ces graves.

- Les contraintes sur les facettes verticales sont concentrées immédiatement sous la tête de la colonne et à l'interface limon-graves (fig. 13).

- Les déplacements verticaux sont, en tête de colonne, de $3,5 \mathrm{~mm}$, ils deviennent négligeables à une distance de $3 \mathrm{~m}$ de l'axe de la colonne (fig. 14).

Les déplacements horizontaux ont une forme «en tonneau " dans le limon. Ces déplacements s'accroissent lorsqu'on s'éloigne de la colonne, ceci est dâ à ce que le coefficient de Poisśon a été pris égal à 0,2 dans la colonne et 0,5 dans le limon. Ces déplacements sont au maximum de $0,5 \mathrm{~mm}$ (fig. 15).

- Les éléments qui entrent en plasticité sont les éléments de la colonne traversant la couche de limon. Ils apparaissent pour une charge de $250 \mathrm{kN}$. Ces éléments sont en effet soumis à des contraintes verticales très élevées et des contraintes horizontales faibles (fig. 16 et 17).

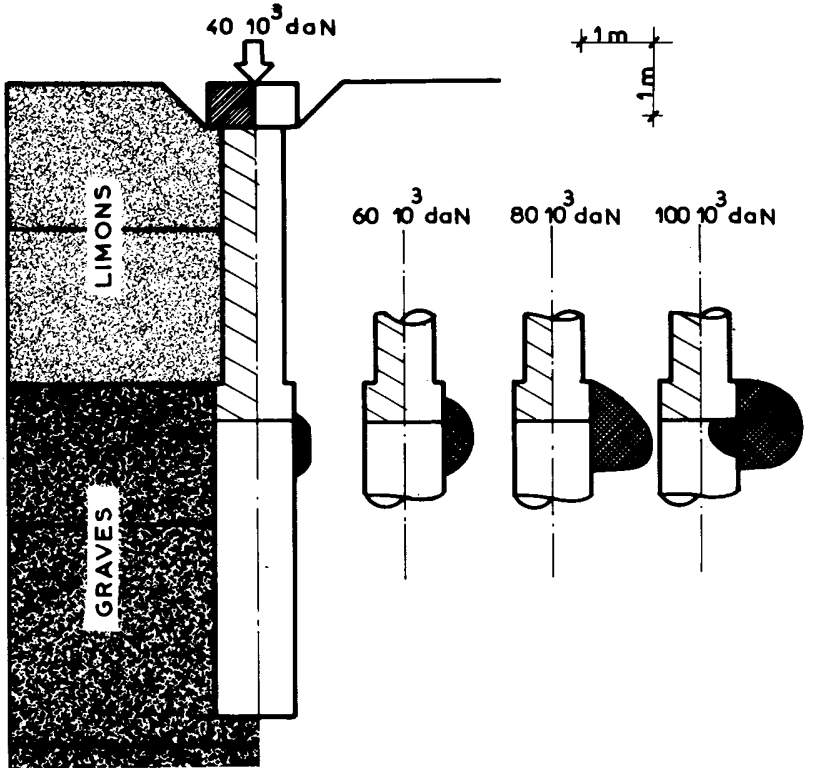

Fig. 23 Colonne cimentée - Calcul élasto-plastique Développement des zones à l'état limite plastique sous charge variant de 40 à $100.10^{3}$ daN

\subsubsection{Colonne ballastée cimentée chargée à $400 \mathrm{kN}$}

Les mêmes remarques peuvent être faites en ce qui concerne la colonne cimentée. Le déplacement vertical en tête est de $5,9 \mathrm{~mm}$ (fig. 21), le déplacement horizontal maximum dans le limon est. de $0,9 \mathrm{~mm}$ (fig. 22). Les premiers éléments qui entrent en plasticité sont situés dans les graves au voisinage de la colonne. Ils apparaissent pour une charge de $400 \mathrm{kN}$ (fig. 23). En effet, les éléments de la colonne cimentée ayant une cohésion importante n'entrent pas en plasticité, par contre dès que la colonne pénètre dans les graves, il y a report de contraintes important sur ces graves de cohésion très faible qui entrent en plasticité (fig. 23). Lorsque la charge appliquée atteint $1000 \mathrm{kN}(100 \mathrm{t})$ la zone plastique se propage à l'intérieur de la colonne dans la zone non cimentée (fig. 23).

II faut souligner que la mise en plasticité des éléments des deux types de colonnes est très sensible à la valeur de la cohésion adoptée pour le matériau constituant ces colonnes.

\subsection{Comparaison entre mesures in-situ et calculs}

- Contraintes :

Au niveau de la sous face de la semelle, les contraintes calculées apparaissent beaucoup plus faibles à l'interface semelle/sol qu'à l'interface semelle/colonne. L'ordre de grandeur est le suivant, les charges en tête étant, rappelons-le de $250 \mathrm{kN}$ pour la colonne standard et de $400 \mathrm{kN}$ pour la colonne cimentée :

contrainte sur colonne $=.6$ pour colonne standard contrainte sur sol $=7.5$ pour colonne cimentée

La différence qui apparaît entre les deux types de colonnes est qualitativement logique. Les valeurs des taux de répartition calculés sont 2 à 4 fois plus élevées que celles estimées d'après les mesures in-situ. Cet écart peut être imputé à la présence d'une couche de limon nettement plus raide au-dessus de la nappe, dont on n'a pas tenu compte dans les calculs.

En profondeur les contraintes sur les facettes horizontales sont presqu'entièrement concentrées dans la section de la colonne, tant que l'on est dans les limons. Une diffusion dans le terrain ambiant se manifeste dès la pénétration dans les graves.

Les accroissements de contrainte dus à l'application de la charge, sur les facettes verticales à la périphérie de la colonne sont très faibles. Ceci tendrait à prouver que l'absence de réaction notée sur les cellules de mesure 
placées en profondeur à la périphérie de la colonne d'essai, peut n'être pas imputable à une défectuosité métrologique, et bien au contrainre témoigner de la faible importance de la sollicitation.

Il serait intéressant, dans une phase future des investigations par le calcul, d'observer le gain que l'on peut attendre d'une augmentation de la surface de la semelle, cette augmentation ayant vraisemblablement pour effet d'augmenter la contrainte autour de la colonne et par là même de repousser la charge limite engendrant des déviateurs de rupture au sein de la colonne.

Dans le même ordre d'idée on est en droit de supposer que l'effet de groupe joụe un rôle favorable analogue, les colonnes s'épaulant mutuellement. Une telle analyse semble toutefois difficilement accessible au calcul par la méthode des éléments finis, en raison de l'absence de symétrie de révolution du cas de figure.

\section{- Tassements:}

Les résultats des calculs donnent des valeurs absolues de tassement légèrement plus élevées que les mesures.

On obtient un rapport :

$$
\frac{\text { Tassement calculé }}{\text { Tassement } \text { mesuré }}=1.25
$$

Compte tenu des approximations faites au niveau des caractéristiques rhéologiques la concordance paraît satisfaisante.

Les très faibles déplacements radiaux calculés expliqueraient l'absence de résultats observée dans les mesures inclinométriques. 